African Crop Science Journal by African Crop Science Society is licensed under a Creative Commons Attribution 3.0 Uganda License. Based on a work at www.ajol.info/ and www.bioline.org.br/cs DOI: http://dx.doi.org/10.4314/acsj.v25i3.2

\title{
DETERMINISME DE LA STABILITE STRUCTURALE DES SOLS CULTIVES DE LA ZONE COTONNIERE OUEST DU BURKINA FASO
}

\author{
B. OUATTARA, K. OUATTARA, P.J.A. COULIBALY, F. LOMPO, A. YAO-KOUAMÉ ${ }^{1}$
}

et M.P. SÉDOGO

Institut de l'Environnement et de Recherches Agricoles (INERA), 04 BP8645, Ouagadougou 04, Burkina Faso ${ }^{1}$ Université de Côte d'Ivoire, 22 BP 582 Abidjan 22, Côte d'Ivoire

Auteur correspondant: alwatdior58@gmail.com

(Received 31 May, 2017; accepted 7 August, 2017)

\section{RÉSUME}

Une analyse diagnostique synchronique a été conduite pour évaluer l'évolution des états structuraux des sols cultivés de la zone cotonnière Ouest du Burkina Faso. Des échantillons de sol ont été prélevés sur les horizons de surface $(0-15 \mathrm{~cm})$ de 101 parcelles, à partir d'une typologie des grands systèmes de cultures (itinérante, cyclique et permanente) et d'intensité de travail du sol (labour occasionnel, bisannuel, annuel). Les analyses ont porté sur les teneurs en agrégats stables, argiles, polycations et composés organiques des sols. Les teneurs en composés organiques du sol ont été significativement influencées par les systèmes de culture et corrélées aux taux d'agrégats stables. Les sucres extraits à l'eau chaude ont expliqué $35 \%(\mathrm{P}<0,001)$ de la variabilité de la stabilité structurale des sols, contre $25 \%$ pour le carbone total. Parmi les constituants minéraux du sol, l'argile et les oxyhydroxydes de fer sous leur forme amorphe ont respectivement contrôlé $53 \%(\mathrm{P}<0,001)$ et $40 \%(\mathrm{P}<0,001)$ de l'agrégation des sols dans les champs et jachères confondus. La part agrégative du calcium n'a été que de $17 \%(\mathrm{P}<0,01)$. Toutefois, l'influence de chacun de ces constituants minéraux a été davantage renforcée (près de $70 \%, \mathrm{P}<0,001$ ) dans les jachères naturelles. Leur action agrégative y atteignait $80 \%(\mathrm{P}<0,001)$ lorsqu'ils étaient associés au carbone soluble ou au carbone de la biomasse microbienne du sol. Il a été ainsi mis en exergue, la nécessité de maintenir une couverture végétale vivante à la surface de ces sols en vue d'affermir leur état structural.

Mots Clés: Agrégats, calcium, jachère naturelle, matière organique, oxyhydroxides de fer

\begin{abstract}
A synchronic diagnosis analysis was conducted to evaluate cultivated soil structural status in the western cotton cultivating area of Burkina Faso. Soils from 101 plots were sampled from the surface layers $(0-15 \mathrm{~cm})$, based on a typology of major cropping systems (shifting, cyclic, continuous cultivation) and tillage intensity (occasional, biennial, annual ploughing). Laboratory analyses were performed on soil water stable aggregates, clay, polycations and organic components content. Soil organic components were significantly influenced by the cropping systems. Organic carbon extracted with hot water explained $35 \%(\mathrm{P}<0.001)$ of the variability of soil structural stability, against $25 \%$ for the total carbon. Among the inorganic soil constituents, clay and iron oxyhydroxides in their amorphous form were controlling respectively $53 \%(\mathrm{P}<0.001)$ and $40 \%(\mathrm{P}<0.001)$ the soil aggregation variance under fields and fallow lands taken in account. The aggregative contribution of calcium was only $17 \%(\mathrm{P}<0.01)$. However, the induced-effect of each of these inorganic components was more strengthened $(70 \%, \mathrm{P}<0.001)$ under natural fallow lands. Their aggregative action reached $80 \%(\mathrm{P}<0.001)$ when associated with hot-water soluble carbon or soil microbial biomass carbon. The results highlighted the need to maintain a plant cover on the surface of tropical soils to consolidate their structural stability.
\end{abstract}

Key Words: Aggregates, calcium, iron oxyhydroxides, natural fallow, organic matter 


\section{INTRODUCTION}

L'état physique du sol agricole constitue l'un des piliers qui sous-tendent sa capacité productive (Boiffin et Marin-Laflèche, 1990 ; Ouattara et al., 2011). En effet, le sol agricole est un édifice construit à partir d'agrégats de différentes tailles qui résultent de l'assemblage des particules organiques et minérales (Oades, 1984). Ces agrégats ménagent entre eux un espace poral qui joue un rôle déterminant dans la circulation de l'eau et de l'air, voire le stockage et la disponibilité des nutriments (Wahl et al., 2004, Ouattara et al., 2007). La nature et l'intensité des liaisons entre les différentes particules d'une part, et entre les différents agrégats d'autre part, confèrent à l'architecture du sol une résistance plus ou moins grande lorsque celui-ci est soumis à des sollicitations extérieures telles que les pratiques culturales (Ouattara et al., 2011). Le « liant» entre les particules et/ou entre les agrégats du sol peut être de nature minérale. C'est le cas du rôle joué par les argiles, les oxyhydroxydes de fer, les polycations, etc. (Zhao et al., 2017). Ce ciment peut également être de nature organique. Dans ce cas, l'agent liant n'est autre que l'une des formes de la matière organique présente dans le sol. Il s'agit, entre autres, des chevelus racinaires, de l'humus, des sucres, du carbone de la biomasse microbienne morte, des mucilages, etc. (Blankinship et al., 2016 ; Sall et al., 2016).

De nombreuses études indiquent que la matière organique constitue le pivot de la fertilité des sols cultivés, en ce sens qu'elle gouverne en grande partie l'ensemble de leurs propriétés physiques, biologiques et chimiques. Elle favorise ainsi le recyclage, le stockage et la disponibilité des éléments nutritifs au profit des plantes, à travers l'amélioration de la capacité d'échange cationique (Tessier et al., 1999). Elle intervient aussi comme source de nutriments et d'énergie pour les microorganismes et la macro faune du sol (Sall et al., 2016). En jouant le rôle de «liant organique » dans les sols, elle contribue ainsi à l'amélioration de leur état structural (Oades, 1984 ; Ouattara et al., 2011).

Des travaux menés en Afrique SudSaharienne ont montré que la mise en culture des sols s'accompagne inexorablement d'un abaissement important du statut organique (Ouattara et al, 2006 ; Srinivasarao et al., 2014). Cette baisse, beaucoup plus accentuée au niveau du pool de carbone labile (matière organique particulaire, carbohydrates, carbone de la biomasse microbienne), se manifeste plus au cours des 5 à 10 premières années de culture (3 à $5 \%$ de perte annuelle) pour ensuite s'équilibrer (Sédogo et al., 1994; Balesdent et al., 2000; Ouattara et al., 2006; Ouattara et al., 2011). Elle s'accompagne d'une décroissance de la stabilité structurale du sol (Ouattara et al., 2011). D'autres travaux ont montré que la déstructuration du sol peut également être due à une perte de la fertilité chimique du sol, notamment la perte en poly cations sous les effets induits des pratiques culturales (Duval, 1993 ; Igwe and Nwokocha, 2006).

En somme, le phénomène d'agrégation ou de dégradation de la structure des sols peut provenir de la fertilité originelle (sol sous jachère) ou de la fertilité acquise du sol, résultant des effets des pratiques culturales. Les effets de la matière organique sur l'état structural des sols sont relativement bien connus dans les sols tempérés (Tisdall et Oades, Bottinelli et al., 2017) ; cependant, on dispose en revanche de peu d'informations sur son rôle dans la fertilité physique des sols tropicaux des régions semi-arides.

C'est dans cette optique qu'il faut situer la présente étude, qui se propose de mieux comprendre les mécanismes qui contrôlent les phénomènes d'agrégation ou de dégradation de la structure du sol en fonction des systèmes de culture. Pour ce faire, les investigations se sont appuyées sur des parcelles paysannes dans le terroir de Bondoukui, situé dans la zone cotonnière Ouest du Burkina Faso. Celui-ci 
offre des situations assez contrastées en matière de paysages agricoles (cultures, jachères) et d'intensification de l'agriculture (cultures manuelle, attelée bovine, motorisée). Aussi, il s'agit, à travers l'établissement de corrélations mathématiques entre les paramètres physico-chimiques et biologiques des sols, d'une part, et le taux d'agrégats stables à l'eau, d'autre part, de statuer sur le déterminisme de la stabilité structurale de ces sols. On pourra ainsi dégager des éléments pertinents d'aide à la décision, permettant d'asseoir des référentiels techniques pour une gestion durable des terres agricoles dans les agrosystèmes cotonniers du Burkina Faso.

\section{MATERIELS ET METHODES}

Site d'étude. L'étude a été réalisée à Bondoukui $\left(11^{\circ} 51^{\prime} \mathrm{N}, 3^{\circ} 46^{\prime} \mathrm{W}, 360 \mathrm{~m}\right.$ d'altitude) en 2009, dans la zone cotonnière Ouest du Burkina Faso. La pluviométrie annuelle moyenne se situe entre 900 et 1000 $\mathrm{mm}$ et est distribuée, de façon monomodale, entre Mai et Octobre. Les températures maxima trainent entre $31^{\circ} \mathrm{C}$ et $30^{\circ} \mathrm{C}$ (Ouattara et al., 2011). L'évapotranspiration potentielle annuelle atteint en moyenne $1900 \mathrm{~mm}$. Selon la classification de FAO (2006), les sols appartiennent à l'ordre des Lixisol ferriques (texture sablo-limoneuse) et des Luvisol ferriques (texture limono argileuse à argileuse) . Les principales caractéristiques physicochimiques de ces sols qui s'apparentent aux sols ferrugineux tropicaux (C.P.C.S., 1967) sont présentées dans le Tableau 1.

Méthode d'échantillonnage. L'échantillonnage du sol a été effectué à l'échelle de la parcelle. Celle-ci se définit comme étant un espace de physionomie et d'histoire culturale homogènes. Le choix des parcelles a été fait à partir d'une typologie des exploitations agricoles en fonction de l'intensité culturale. Trois grands systèmes de cultures (GSC) ont été ainsi identifiés : (i) le système de culture itinérante, caractérisé par 5 à 10 ans de culture suivi de 30 ans de jachère. Ces vieilles jachères sont localement appelées diuré;

(ii) le système de culture à jachère ou de culture cyclique, caractérisé par 5 à 10 ans de culture, suivie de 5 à 20 ans de jachère;

(iii) le système de culture permanente, avec plus de 20 ans de cultures continues.

Ces GSC ont été recoupés avec l'âge des phases culture-jachère, le régime du travail du sol et la texture (Tableau 2). Certaines parcelles du système de culture permanente ont reçu des apports de matières organiques sous forme de déjections animales ou de compost. Les doses ont varié entre 2 et 5 t.ha $^{-1}$, à des fréquences d'application également variables (2 à 3 ans).

Les labours ont été réalisés, en culture attelée ou motorisée, à des profondeurs de plus $15 \mathrm{~cm}$.

Au total, les sols de 101 parcelles (Lot A), ont été échantillonnés pendant la saison sèche en vue d'éviter les risques d'évolution de leur composition chimique. Par ailleurs, un second sous lot B de 33 parcelles a été également

TABLEAU 1. Caractéristiques physico-chimiques des sols sous jachères naturelles

\begin{tabular}{lr}
\hline Caractéristiques & Valeurs $(\mathrm{N}=33)$ \\
\hline Argiles + limons $\left({\left.\mathrm{g} . \mathrm{kg}^{-1}\right)}^{-1}\right)$ & $266 \pm 115$ \\
Sables totaux $\left(\mathrm{g}_{\mathrm{kg}}\right)$ & $544 \pm 141$ \\
Densité apparente $\left(\mathrm{kg} \mathrm{dm}^{-3}\right)$ & $1,48 \pm 0,08$ \\
Somme des bases $\left(\mathrm{cmol} \mathrm{kg}^{-1}\right)$ & $3,30 \pm 1,7$ \\
$\mathrm{CEC}(\mathrm{cmol} \mathrm{kg})$ & $3,70 \pm 2,2$ \\
Carbone organique $\left(\mathrm{g} \mathrm{kg}^{-1}\right)$ & $6,01 \pm 1,6$ \\
Azote $\left(\mathrm{mg} \mathrm{kg}^{-1}\right)$ & $0,41 \pm 0,1$ \\
$\mathrm{pH}$ & $6,20 \pm 0,3$ \\
\hline
\end{tabular}

$\mathrm{CEC}=$ Capacité d'échange cationique; $\mathrm{N}=$ Nombre de parcelles échantillonnées. Valeurs affectées des écarttypes 
TABLEAU 2. Répartition des parcelles entre les différents systèmes de culture

\begin{tabular}{|c|c|c|c|c|c|c|c|c|}
\hline & \multicolumn{2}{|c|}{ Système Itinérant } & \multicolumn{3}{|c|}{ Système à jachère } & \multicolumn{3}{|c|}{ Système permanent } \\
\hline & $\mathrm{J} 30$ & $\mathrm{C} 10$ & $\mathrm{~J} 10$ & $\mathrm{~J} 20$ & $\mathrm{C} 10$ & $\mathrm{Lab} / 2 \mathrm{an}$ & Lab./an & Fumier \\
\hline N. Parcelles A & 11 & 9 & 9 & 13 & 22 & 16 & 15 & 6 \\
\hline N. Parcelles B & 5 & 4 & 4 & 5 & 5 & 5 & 5 & - \\
\hline
\end{tabular}

J30 (Jachère de 30 à 40 ans); J10 (Jachère de 1 à 10 ans) ; J20 (Jachère de 11 à 20 ans); C10 (Culture de 1 à 10 ans); Lab/2an (Labour bisannuel); Lab/an (labour annuel); N. Parcelles A (Nombre de parcelles du lot A) ; N. Parcelle B (Nombre de parcelle du lot B)

constitué en vue d'y réaliser les analyses de sol plus lourdes et plus coûteuses. Le Tableau 2 résume la typologie des parcelles retenues selon les besoins de l'étude.

Les prélèvements ont été réalisés à la faveur des mesures de densité apparente sèche du sol, à l'aide du densitomètre à membrane, sur une profondeur de $15 \mathrm{~cm}$, et à raison de 3 répétitions par parcelle. L'emplacement du point de prélèvement a été déterminé à partir du jet d'un cerceau. Le volume de sol prélevé au densitomètre à membrane était de l'ordre du décimètre cube. Les trois prélèvements ont été ensuite mélangés pour constituer un échantillon composite.

Méthodes d'analyse. La stabilité structurale a été déterminée à travers la mesure du taux d'agrégats stables du sol de taille supérieure à $200 \mu \mathrm{m}$, après un tamisage standardisé dans l'eau, pendant une heure. C'est une technique adaptée aux sols sableux, mise au point par Bloin et al. (1990) et qui était, en réalité, une variante de celle préconisée par Kemper et Rosenau (1986). La teneur en agrégats stables, après une heure de tamisage dans l'eau (Ag1h), a été exprimée en pourcentage du poids du sol total, à l'aide de formule :

$$
\mathrm{A} g 1 h=100 \frac{\text { Tamisat }- \text { Sables Grossiers }}{\text { Prise d'essai }- \text { Sables Grossiers }}
$$

Le tamisat est constitué d'agrégats organominéraux et de sables de taille supérieure à 200 $\mu \mathrm{m}$.
La teneur en carbone organique totale du sol ( $\left.\mathrm{g}-\mathrm{C} \cdot \mathrm{kg}^{-1}-\mathrm{Sol}\right)$ a été dosée selon la méthode de Walkley et Black, adaptée aux sols du Burkina par Gnankambary et al. (1999).

Le carbone soluble dans l'eau a été extrait et dosé selon la méthode décrite par Bachelier (1966).

Les sucres monomères ont été extraits et dosés dans le laboratoire d'Analyses AgroAlimentaires du Centre International de Recherche Agronomique pour le Développement en coopération (CIRAD) à Montpellier, en utilisant la méthode préconisée par Oades et al. (1970).

Le carbone de la biomasse microbienne a été estimé selon la méthode (Jenkinson et Powlson, 1976) à partir des échantillons de sols fumigés et non fumigés. Le fumigant utilisé était du chloroforme débarrassé de l'éthanol après plusieurs lavages à l'eau.

Pour accéder aux formes de fer «libres » cristallisées ou amorphes, deux modes d'extraction sélectives ont été utilisées en s'inspirant des protocoles décrits par Rouiller et al. (1994).

La capacité d'échange cationique (CEC) et les bases échangeables du sol ont été dosées au laboratoire Matière Organique des Sols Tropicaux (MOST) du CIRAD. Le complexe d'échange du sol a été analysé en utilisant comme ion échangeur le chlorure de cobaltihexamine, selon la méthode décrite par Fallavier et al. (1985). La détermination de la CEC effective du sol (quantifiée au pH du sol) a été utilisée, car elle permet d'approcher au mieux la capacité d'échange du sol en place. 
Elle constitue un bon outil du suivi de la qualité physique et chimique des sols.

Les teneurs en argiles des sols ont été déterminées selon la méthode internationale adaptée à la pipette de Robinson Khöln. Elle a été réalisée sur la terre fine (tamisat de $2 \mathrm{~mm}$ ) après une destruction de la matière organique à l'eau oxygénée et une dispersion des argiles à l'aide de l'hexamétaphosphate de sodium (HMP).

Les analyses de variance et la détermination des caractéristiques des équations de régression ont été réalisées à l'aide du logiciel Genstat $6^{\text {ème }}$ édition.

\section{RESULTATS}

Variation des déterminants de l'agrégation en fonction des systèmes de culture. Les teneurs de quelques constituants chimiques et biologiques des sols résultant des effets induits des pratiques culturales sont présentés dans le Tableau 3. Les teneurs des constituants organiques se sont différenciées de façon très significative entre les jachères et les champs cultivés. Par contre, les teneurs du sol en éléments minéraux n'ont pas significativement varié en fonction des systèmes de culture.

Déterminants minéraux de l'agrégation. Le Tableau 4 a récapitulé l'ensemble des caractéristiques des équations de régression linéaire simple relatives aux déterminants minéraux et organiques de l'agrégation mesurés dans les champs et jachères confondus. Les résultats ont montré que le fer amorphe expliquait près de $40 \%$ la variabilité de la stabilité structurale du sol. Cette action bénéfique était cependant précédée de celle de l'argile, qui a contrôlé plus de la moitié (53\%) de la variabilité de l'agrégation du sol. La forme de fer cristallisée, quant à elle, n'a pas influencé, de façon significative, la stabilité structurale. La capacité d'échange cationique du sol a contrôlé, quant à elle, $26 \%$ de l'agrégation.

De l'ensemble des bases échangeables du sol, seul le calcium a affiché un effet significatif dans le contrôle de la stabilité structurale. La variance expliquée a été de 17 $\%$ contre $23 \%$ pour l'ensemble des bases échangeables.

\section{Déterminants organiques de l'agrégation.}

L'action agrégative des matières organiques a varié selon leurs formes dans le sol (Tableau 4). Le carbone soluble à l'eau chaude a expliqué $35 \%$ de la variance de la stabilité structurale du sol. Mais, au-delà des teneurs de $60 \mathrm{mg}$ C.kg-sol, l'augmentation de la concentration en carbone soluble n'a plus été accompagnée d'un accroissement de la stabilité structurale des sols (Fig. 1). Par contre, le carbone organique total n'a contrôlé

TABLEAU 3. Teneurs de quelques paramètres physico-chimiques et biologiques des sols

\begin{tabular}{|c|c|c|c|c|}
\hline & Champs & Jachère & LSD & $\mathrm{P}$ \\
\hline CEC $\left(\mathrm{cmol} \cdot \mathrm{kg}^{-1}\right)$ & 3,4 & 3,7 & - & - \\
\hline $\mathrm{Ca}^{2+}\left(\mathrm{cmol} \cdot \mathrm{kg}^{-1}\right)$ & 2,25 & 2,18 & - & - \\
\hline Fer cristallisé $_{200 \mu \mathrm{m}}\left(\mathrm{mg} \mathrm{kg}^{-1}\right)$ & 0,307 & 0,349 & - & - \\
\hline Fer amorphe ${ }_{200 \mu \mathrm{m}}\left(\mathrm{mg} \mathrm{kg}^{-1}\right)$ & 0,154 & 0,157 & - & - \\
\hline Carbone total (g-C.kg $\left.{ }^{-1}-\mathrm{Sol}\right)$ & 4,9 & 6,6 & 0,8 & $<0,001$ \\
\hline Carbone soluble (mg-C. $\left.\mathrm{kg}^{-1}-\mathrm{Sol}\right)$ & 37,4 & 45,3 & 5,2 & 0,004 \\
\hline Polysaccaharides (mg-C.kg-1-Sol) & 320,0 & 515,0 & 37,0 & 0,006 \\
\hline Carbone biomasse (mg-C.kg-1-Sol) & 7,5 & 12,5 & 2,3 & $<0,001$ \\
\hline
\end{tabular}

$\mathrm{P}=$ Seuil de signification, $\mathrm{CEC}=$ Capacité d'échange cationique, $\mathrm{Ca}^{2+}=$ Calcium, Fer cristallisé ${ }_{200 u m}=$ Fer extrait au réactif de Mehra-Jackson sur la fraction de sol $<200 \mu \mathrm{m}$, Fer amorphe ${ }_{200 \mu \mathrm{m}}=$ Fer extrait au réactif de Tamm sur la fraction de sol $<200 \mu \mathrm{m}$, Carbone soluble (Carbone organique extrait à l'eau chaude, biomasse microbienne $=$ Carbone de la biomasse microbienne du sol 
TABLEAU 4. Déterminants de l'agrégation (Champs et jachères confondus) et caractéristiques des équations de régression simple

\begin{tabular}{|c|c|c|c|c|}
\hline \multirow[t]{2}{*}{ Déterminants } & \multicolumn{2}{|c|}{ Qualité de la régression } & \multicolumn{2}{|c|}{ Coefficients de la régression } \\
\hline & Variance expliquée (\%.) & $\mathrm{P}$ & $\mathrm{a}$ & $\mathrm{b}$ \\
\hline Argile & 53 & $<0,001$ & 0,80 & - \\
\hline Fer amorphe ${ }_{200 \mathrm{~mm}}$ & 40 & $<0,001$ & 140,2 & $-10,7$ \\
\hline $\mathrm{CEC}$ & 26 & 0,002 & 2,03 & 3,73 \\
\hline Bases échangeables & 23 & $<0,001$ & 3,21 & - \\
\hline $\mathrm{Ca}^{++}$ & 17 & 0,009 & 2,90 & 4,50 \\
\hline Carbone total & 25 & $<0,001$ & 1,93 & - \\
\hline Carbone soluble & 35 & $<0,001$ & 0,25 & - \\
\hline Polysaccharides & 13 & 0,02 & 4,39 & 6,58 \\
\hline Galactose & 17 & 0,01 & 38,0 & 6,74 \\
\hline Mannose & 24 & 0,002 & 42,6 & 6,89 \\
\hline Galactose, Mannose & 25 & 0,002 & 24,55 & 5,90 \\
\hline Biomasse microbienne & 20 & $<0,001$ & 1,08 & - \\
\hline
\end{tabular}

$\mathrm{P}=$ Seuil de signification, $\mathrm{CEC}=$ Capacité d'échange cationique, $\mathrm{Ca}^{++}=$Calcium, Fer amorphe ${ }_{200 u m}=$ Fer extrait au réactif de Tamm sur la fraction de sol $<200 \mu \mathrm{m}$, Carbone soluble $=$ Carbone organique extrait à l' eau chaude, Biomasse microbienne $=$ Carbone de la biomasse microbienne du sol

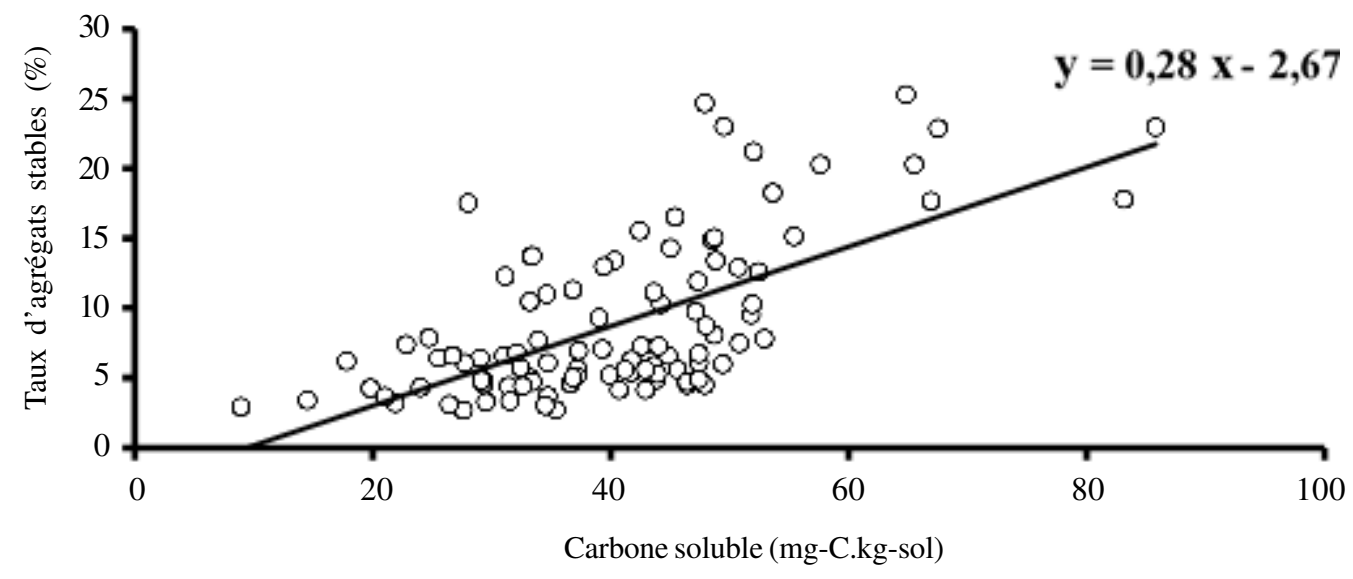

Figure 1. Relation entre le taux d'agrégats stables à l'eau et les teneurs en carbone soluble du sol.

que $25 \%$ de l'agrégation du sol. Son action agrégative a été beaucoup plus lâche comparativement à celle du carbone soluble (Fig. 2).

La relation entre les polysaccharides totaux (somme des sucres monomères) et le taux d'agrégats stables a été très lâche $\left(\mathrm{R}^{2}=0,13\right)$. Mais, la somme galactose + mannose a pu expliquer le quart de la variabilité de l'agrégation (25\%.). Toutefois, cette action a été équivalente à celle induite par le mannose seul $(24 \%$.), bien que le galactose ait expliqué $17 \%$ de la variance du taux d'agrégats stables à l'eau.

Le carbone de la biomasse microbienne a contrôlé environ $20 \%$ de la variance de l'agrégation. Cette action agrégative a été davantage renforcée $\left(\mathrm{R}^{2}=0,35 ; \mathrm{P}<0,001\right)$ 


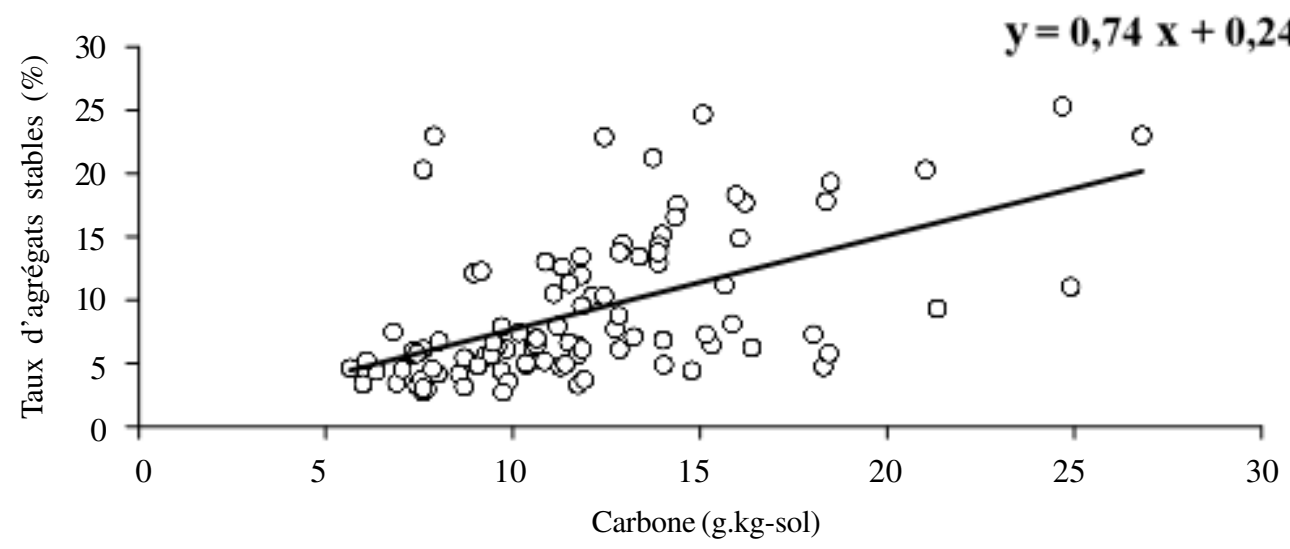

Figure 2. Relation entre le taux d'agrégats stables à l'eau et les teneurs en carbone organique du sol.

dans les sols sous jachère naturelle (Tableau $4)$.

Actions combinées des déterminants de l'agrégation. Les actions combinées des différents déterminants chimiques et biologiques de l'agrégation du sol ont été mises en exergue à partir des équations de régression multiple dont les caractéristiques sont consignées dans le Tableau 5.

Comme indiqué dans le Tableau 4, l'argile a expliqué, à elle seule, plus de la moitié (53 $\%)$ de la variance de l'agrégation. Elle a été suivie du fer amorphe, (40\%). Toutefois, l'action de l'argile, combinée à celle du fer, n'est parvenue à expliquer que $57 \%$ de la variabilité de la stabilité structurale (Tableau 5). Mais, lorsque l'action de l'argile fut associée à celle de l'une des fractions du carbone organique du sol, la qualité de la régression a augmenté, de façon significative, à $66 \%$, avec le carbone soluble et à $60 \%$, avec le mannose.

De même, le carbone soluble, associé au fer amorphe, a amélioré la qualité de la régression $(59 \%$.). On pourra également noter, dans le Tableau 5, que les parts de la variance de l'agrégation expliquées par les associations (argile + carbone soluble, $\mathrm{R}^{2}=$ $0,66)$ d'une part, (carbone soluble + fer amorphe, $\left.R^{2}=0,59\right)$ d'autre part, n'ont augmenté que de trois points lorsqu'on leur a associé respectivement, le fer amorphe et la CEC.

La CEC s'est effectivement révélée être un facteur déterminant de l'agrégation du sol, surtout lorsqu'elle a été associée au carbone soluble : la variance expliquée a été de $55 \%$. En revanche, la contribution du carbone de la biomasse microbienne du sol, associée à l'un ou l'autre des constituants minéraux du sol, n'a guère amélioré la qualité des régressions.

Actions agrégatives des différents agents sous jachères naturelles. Lorsque l'action des différents facteurs de l'agrégation des sols est analysée sous jachère naturelle, il en est résulté une nette amélioration de la qualité des régressions (Tableau 6). Ainsi, le calcium et la CEC ont chacun contrôlé plus de $60 \%$ de la variance de la stabilité structurale. Mais leurs actions combinées sont restées en deçà de leurs performances individuelles. Par contre, elles ont été davantage renforcées en présence de composés organiques (carbone soluble, carbone de la biomasse microbienne). Ainsi, le carbone soluble, associé au calcium ou à la CEC, a expliqué près de $80 \%$ de la variance de l'agrégation des sols. A titre illustratif, il est montré sur la Figure 3 que le taux d'agrégats stables a augmenté de façon exponentielle avec les teneurs croissantes du sol en calcium. 
TABLEAU 5. Déterminants de l'agrégation (champs et jachères confondus) et caractéristiques des équations de régression multiple

\begin{tabular}{|c|c|c|c|c|c|c|}
\hline \multirow[t]{2}{*}{ Déterminants } & \multicolumn{2}{|c|}{ Qualité de la régression } & \multicolumn{4}{|c|}{ Coefficients de la régression } \\
\hline & $\begin{array}{c}\text { Variance } \\
\text { expliquée }(\%)\end{array}$ & $\mathrm{P}$ & $\mathrm{a}$ & $\mathrm{b}$ & $\mathrm{d}$ & Constante \\
\hline Argile, Somme des bases & 53 & $<0,001$ & 0,65 & 0,64 & - & - \\
\hline Argile, $\mathrm{Fer}_{\mathrm{T}}$ & 57 & $<0,001$ & 0,54 & 69,7 & - & $-7,11$ \\
\hline Argile, Mannose & 60 & $<0,001$ & 0,63 & 25,66 & - & - \\
\hline Argile, Csoluble & 66 & $<0,001$ & 0,61 & 0,19 & - & $-5,31$ \\
\hline Carbone total, CEC & 37 & $<0,001$ & 1,06 & 1,44 & - & - \\
\hline C. soluble, CEC & 55 & $<0,001$ & 0,29 & 1,76 & - & $-7,88$ \\
\hline C. soluble, Fer $_{T}$ & 59 & $<0,001$ & 0,25 & 108,1 & - & 16,73 \\
\hline Argile, C. soluble, Fer $_{T}$ & 69 & $<0,001$ & 0,44 & 0,18 & 56,8 & $-11,8$ \\
\hline C. soluble, $\mathrm{CEC}, \mathrm{Fer}_{\mathrm{T}}{ }^{\mathrm{T}}$ & 62 & $<0,001$ & 0,25 & 0,98 & 77,4 & $-15,45$ \\
\hline
\end{tabular}

$\mathrm{P}=$ Seuil de signification, FerT $=$ Fer extrait au réactif de Tamm sur la fraction de sol $<200 \mu \mathrm{m}$, C.soluble $=$ Carbone soluble dans l'eau chaude, $\mathrm{CEC}=$ Capacité d'échange cationique

TABLEAU 6. Déterminants de l'agrégation dans les sols sous jachères naturelles et caractéristiques des équations de régression multiple

\begin{tabular}{|c|c|c|c|c|c|}
\hline \multirow[t]{2}{*}{ Déterminants } & \multicolumn{2}{|c|}{ Qualité de la régression } & \multicolumn{3}{|c|}{ Coefficients de la régression } \\
\hline & Variance expliquée (\%) & $\mathrm{P}$ & a & $\mathrm{b}$ & Constante \\
\hline Argile & 72 & $<0,001$ & 0,94 & - & - \\
\hline Calcium & 66 & $<0,001$ & 7,70 & - & - \\
\hline CEC & 62 & $<0,001$ & 4,55 & - & - \\
\hline Somme des bases & 61 & $<0,001$ & 4,91 & - & - \\
\hline $\mathrm{Fer}_{\mathrm{T}}$ & 40 & $<0,001$ & 94,8 & - & - \\
\hline Argile, $\mathrm{Fer}_{\mathrm{T}}$ & 58 & $<0,001$ & 0,54 & 43,3 & - \\
\hline Argile, Somme des bases & 68 & $<0,001$ & 0,40 & 2,91 & - \\
\hline Calcium, Biomasse micro. & 70 & 0,001 & 9,31 & 0,35 & $-7,83$ \\
\hline Calcium, C.soluble & 80 & $<0,001$ & 8,97 & 0,16 & $-11,21$ \\
\hline
\end{tabular}

$\mathrm{P}=$ Seuil de signification, $\mathrm{CEC}=$ Capacité d'échange cationique, FerT $=$ Fer extrait au réactif de Tamm sur la fraction de sol $<200 \mu \mathrm{m}$, C. soluble $=$ Carbone soluble dans l'eau chaude, Biomasse micro $=$ Carbone de la biomasse microbienne du sol

\section{DISCUSSION}

Lorsque des agrégats terreux secs sont soumis à l'action du tamisage standardisé dans l'eau, il se produit deux séries de processus physiques qui conditionnent leur stabilité. Il s'agit, d'une part, des mécanismes d'éclatement des agrégats lié au piégeage de l'air lors de l'imbibition, et d'autre part, des phénomènes de désagrégation mécanique due à l'agitation une fois que l'échantillon est saturé (Chenu et al., 2000; Ouattara et al., 2008). De nombreux travaux rapportent la prépondérance des phénomènes d'éclatement (Oades, 1984; Kouakoua et al., 1999 ; Consentino et al., 2006). Quoiqu'il en soit, il est connu que la désagrégation dans l'eau est sous la dépendance étroite des effets des matériaux organiques et 


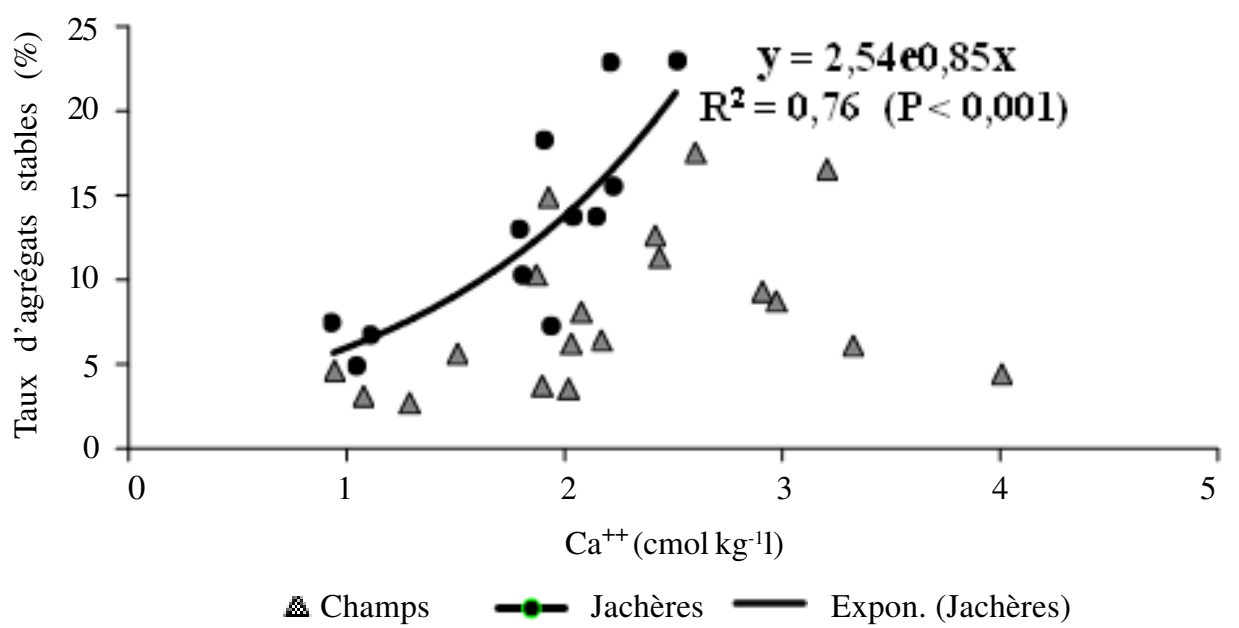

Figure 3. Relation entre les teneurs en calcium des sols et le taux d'agrégats stables à l'eau.

minéraux intrinsèques du sol (Oades, 1984 ; Albrecht et al., 1998).

\section{Importance de l'argile et des poly cations} dans la structuration du sol.

La nature et les propriétés des constituants minéraux des sols déterminent le niveau de base de l'agrégation (Tisdall et Oades, 1982; Albrecht et al., 1998). Les sols de Bondoukui sont du type ferrugineux tropical, riches en sesquioxydes de fer, avec une fraction argileuse dominée par la kaolinite (Ouattara et $a l ., 2011)$; ce type d'argile est connu être chimiquement peu actif dans les sols (Six et $a l .$, 2002). Toutefois, de l'ensemble des constituants inorganiques agrégeants étudiés, l'argile a expliqué, à elle seule, plus de la moitié de la variance de l'agrégation. En effet, Duval et al. (1993), Gelaw et al., 2013), ont rapporté que les argiles, de par leurs propriétés électrostatiques, jouaient un rôle majeur dans la cohésion des sables et des limons, qui sont sans charge électrique.

L'efficacité des argiles sur la stabilité structurale peut être aussi influencée par le système de culture pratiqué. Les résultats obtenus sont en accord avec ceux de Duval et al. (1993) et de Gelaw et al. (2013) qui ont montré que l'effet améliorant des couvertures permanentes et vivantes du sol (prairie ou jachère), sur sa structure, augmentait avec les teneurs croissantes en argile. Elle a, en effet, contrôlé plus de $70 \%$ de la variabilité de l'agrégation dans les sols sous jachères naturelles de Bondoukui.

Dans ces sols riches en sesquioxydes de fer, les résultats ont mis en évidence une corrélation assez étroite et significative entre les macro agrégats stables à l'eau et la forme amorphe du fer. Ils sont en accord avec ceux de Lamar et Bresson (1989), qui avaient montré que l'agrégation était fortement corrélée au fer amorphe dans des sols fersiallitiques d'Algérie. Ils corroborent également ceux de Neufeldt et al. (1999), qui rapportaient que les teneurs en fer amorphe ne sont significativement corrélées aux macro agrégats que dans les argiles des oxisols de cerrados brésiliens. Ces résultats attestent ceux de Ouattara et al. (2008), qui ont mis en évidence, sur le site de Bondoukui, l'existence d'une corrélation étroite entre le taux d'agrégats stables à l'eau et les teneurs en phosphore du sol.

Dans les sols de Bondoukui, le taux d'agrégats stables à l'eau a été significativement corrélé aux teneurs en calcium du sol. Ce lien, assez lâche $\left(R^{2}=0,17\right)$ dans le complexe champ-jachères, s'est davantage affermi dans les jachères $\left(R^{2}=\right.$ 
$0,70)$. Ainsi, à l'instar des oxyhydroxydes de fer, le calcium bivalent peut servir de matériau de cimentation en permettant aux argiles de se maintenir un état floculé. Il permet aussi de lier les particules argileuses du sol et les matières organiques, plus abondantes sous les jachères naturelles, pour former les microagrégats stables et permanents (Six et al., 2004, Throckmorton et al., 2015).

Le calcium, cation bivalent, permet de lier les composés organiques et les argiles du sol, sous forme de ponts cationiques (Oades, 1984). Cette action est alors plus accentuée dans les sols sous jachères naturelles, plus riches en matières organiques (Duval et al., 1993).

Le rôle agrégeant des cations calcium ne saurait être séparé de celui de la CEC qui a contrôlé 26 et $62 \%$ de la variance de l'agrégation respectivement, dans le complexe champ-jachères, et dans les jachères seules. En ce sens, la CEC se veut être un paramètre intégrant «quantité » et «nature » des constituants fins des sols que sont les argiles, les oxyhydroxydes et les matières organiques. Et, tout ce qui pourrait contribuer à augmenter la densité de charge des constituants du sol (nombre de cations échangeables), tendrait à renforcer la stabilité structurale par le biais des liaisons électro statiques entre les surfaces solides, tels que les « ponts calciques ». Ainsi, au-delà de son utilisation traditionnelle comme donnée chimique permettant de raisonner la fertilité des sols, la CEC peut aussi servir d'outil d'interprétation et de prévision des propriétés physiques des sols (Tessier et al., 1999).

Matière organique, pivot de la fertilité physique des sols tropicaux. Dans les conditions agro-écologiques de Bondoukui, la part de l'agrégation des sols, contrôlée par le carbone organique total, a été de $30 \%$. En effet, du point de vue physique, il est connu que les matériaux organiques susceptibles de servir de liens entre les particules minérales sont de tailles variables, s'échelonnant depuis les courtes chaînes d'acides organiques jusqu'aux racines (Amézketa, 1999). Mais, des études conduites aussi bien en régions tempérées (Baldock et al., 1987; Six et al., 2004, Throckmorton et al., 2015) que sous les tropiques (Kouakoua et al., 1999; Neufeldt et al., 1999; Adesodum et al., 2001, Throckmorton et al., 2015) ont montré que les opinions divergent dans le choix des formes véritablement agrégeantes de la matière organique des sols.

Dans cette optique, les travaux d'investigation menés à Bondoukui ont montré que sa stabilité structurale du sol était mieux corrélée aux formes labiles de la matière organique. Le carbone soluble dans l'eau chaude a été la fraction organique labile la mieux corrélée à la stabilité structurale $\left(\mathrm{R}^{2}=\right.$ $0,40)$ sur l'ensemble des 101 parcelles étudiées. Ce résultat est en accord avec ceux de Kouakoua et al. (1999), Il a également été mis en évidence sur le site de Bondoukui l'existence d'une valeur seuil (autour de $60 \mathrm{mg}-\mathrm{C} . \mathrm{kg}^{-1}$ Sol) à partir de laquelle l'accroissement des teneurs en carbone organique n'est plus suivi d'une augmentation de la stabilité structurale. De tels comportements ont été observés par Duval et al. (1993), Adesodum et al. (2001), et seraient dus à l'accroissement de charges négatives à la surface des colloïdes; ce qui favoriserait les phénomènes de dispersion (Tisdall et Oades, 1982).

Les teneurs du sol en polysaccharides totaux ne sont parvenues, par contre, à expliquer que $13 \%$ de la variabilité de l'agrégation des sols de Bondoukui. Cette corrélation est devenue plus forte $\left(R^{2}=0,25\right)$ avec les sucres monomères d'origine microbienne (galactose + mannose), et, plus précisément, celle du mannose. Ce qui amène à dire que les sucres hérités de la synthèse microbienne seraient plus performants dans l'agrégation des sols que ceux d'origine végétale (Tisdall et Oades, 1982; Oades, 1984).

Le taux de macro agrégats stables a été aussi significativement corrélé aux teneurs du sol en carbone de la biomasse microbienne 
$\left(R^{2}=0,20\right)$. De telles relations ont été mises en évidence, de façon plus étroite par Six et Paustian (2013), Throckmorton et al. (2015). La biomasse microbienne a influencé positivement l'agrégation en ce sens qu'elle produit du mucilage, qui joue le rôle de ciment vis-à-vis des particules minérales, tandis que les hyphes fongiques rassemblent les micro agrégats grâce à leur action d'enchevêtrement (Oades, 1984).

Comme nous l'avons déjà montré, l'argile et les oxyhydroxydes de fer amorphe se sont avérés être des agents qui, individuellement, contrôlait le plus la stabilité structurale. Mais, leurs effets combinés ont donné des résultats en deçà de ceux induits par leur association respective avec l'une ou l'autre des formes organiques. Toutes choses qui ont confirmé le rôle primordial de la matière organique dans la stabilisation des agrégats (Throckmorton $e t$ al., 2015). Cette action stabilisatrice des agrégats est expliquée par le rôle de liant des composés organiques essentiellement hérités de l'activité biologique. Il s'agit notamment des mucilages microbiens ou racinaires, des hyphes fongiques, des acides organiques (Tisdall et Oades, 1982 ; Oades, 1984 ; Six et al., 2004).

La matière organique, par son caractère hydrophobe, intervient également dans la stabilisation des agrégats formés (Le Bissonnais, 1988). Elle s'oppose ainsi à l'entrée de l'eau dans les agrégats. Toutefois, les travaux de Godefroy et Jacquin (1975) ont montré que le caractère anti-mouillant de la matière organique serait plus marqué dans les zones tempérées que sous les tropiques. Six et al. (2002) ont rapporté ainsi une plus forte relation entre l'agrégation et le statut organique des sols tempérés, alors que le taux de macro agrégats stables à l'eau demeurait beaucoup plus élevé sous les tropiques. Ils ont expliqué cela par la biodégradabilité plus forte de la matière organique, et par des teneurs plus élevées d'oxyhydroxydes de fer dans les sols tropicaux.
L'action agrégative des constituants minéraux et organiques des sols a été davantage renforcée sous les jachères naturelles (Duval et al., 1993) alors qu' aucune différence n'avait pu être décelée dans les teneurs de ces constituants minéraux entre les sols cultivés et ceux sous jachères naturelles. Cela pourrait s'expliquer par le fait que la grande population microbienne dans la rhizosphère des sols sous jachères naturelles, produirait d'importantes quantités de matériaux organiques (glus, mucilage racinaire, acides organiques) qui, associés aux fines racines des herbacées et aux hyphes myccorhiziens ou fongiques, ont eu des effets de liant et d'enchevêtrement sur les agrégats. Il conviendrait d'ajouter à cela, les effets positifs de la grande population de la macro faune (vers de terre, termites, etc.) des sols non cultivés qui, de par ses activités, contribuerait également à améliorer la stabilité structurale. C'est ainsi que le carbone soluble, associé aux ions calcium ou à la CEC a expliqué près de $80 \%$ de la variance de l'agrégation dans les sols sous jachères naturelles.

En somme, les matières organiques associées aux argiles, oxyhydroxydes de fer et polycations formeraient, un plasma argilohumique qui engloberait le squelette minéral. De telles associations seraient responsables de la micro agrégation des sols tropicaux (Gelaw et al., 2013, Zhao et al., 2017).

\section{CONCLUSION}

les travaux conduits dans cette étude, ont montré que l'agrégation du sol a été contrôlée par les constituants minéraux et organiques. Parmi les constituants minéraux d'origine pédogénétique, l'argile et les oxyhydroxydes de fer amorphe ont développé les plus fortes actions agrégatives. Toutefois, le fait qu'il n'ait pas été possible de déceler de modifications perceptibles dans leurs teneurs au cours des successions culture-jachère, a conduit à donner 
un rôle primordial aux composées organiques dans la stabilisation des agrégats formés. C'est alors que les jachères naturelles, plus riches en composées organiques ont été très déterminantes dans l'agrégation des sols. D'un point de vue qualité, les pools labiles de la matière organiques des sols, représentés par les sucres hydrosolubles et le carbone de la biomasse microbienne ont constitué les agents les plus agrégeants. Leurs actions ont été encore plus fortes dans les jachères naturelles, traduisant ainsi l'importance capitale des couvertures végétales dans la structuration des sols.

\section{REMERCIEMENTS}

Les auteurs adressent leurs sincères remerciements à l'Union Européenne qui a financé ces activités dans le cadre du Projet «Mécanismes d'agrégation des sols tropicaux pauvres en argiles gonflantes». Ils adressent également leurs profondes gratitudes aux Laboratoires «Matière Organique des Sols tropicaux, MOST » et «Analyses Agroalimentaires » du Centre International de Recherche Agronomique pour le Développement en Coopération(CIRAD) pour leurs appréciables contributions dans les analyses de sol.

\section{REFERENCES BIBLIOGRAPHIQUES}

Adesodum, J.K., Mbagwu, J.S.C. and Oti, N. 2001. Structural stability and carbohydrate contents of an ultisol under different management systems. Soil and Tillage Research 60:135-142.

Albrecht, A., Angers, D. A., Beare, M. H. et Blanchart, E. 1998. Déterminants organiques et biologiques de l'agrégation : implications pour la recapitalisation de la fertilité physique des sols tropicaux. Cahiers Agricultures 7 : 357-63

Amézketa, E. 1999. Soil aggregate stability: A review. Journal of Sustainable Agriculture 14:83-151.
Bachelier, G. 1966. Les sucres dans les sols et leur dosage global. Cahier. ORSTOM, Série. Pédologique IV (1):9-22.

Baldock, J.A., Kay, B.D. and Schnitzer, M. 1987. Influence of cropping treatments on the monosaccharides content of the hydrolysates of a soil and its aggregate fractions. Canadian Journal of Soil Science 67:489-499.

Balesdent, J., Chenu, C. and Balabane, M. 2000. Relationship of soil organic matter dynamics to physical protection and tillage. Soil and Tillage Research 53:215-230.

Blankinship, J.C., Fonte, S.J., Six, J. and Schimel, J.P. 2016. Plant versus microbial controls on soil aggregate stability in a seasonally dry ecosystem. Geoderma 272 : 39-50.

Bloin, M., Philippy, R. et Bartoli, F. 1990. Dossier de valorisation d'un prototype de désagrégation des sols. Institut National de la Propriété Industrielle, Paris. 16 p.

Boiffin, J. et Marin-Laflèche, A. 1990. La structure du sol et son évolution: conséquences agronomiques, maîtrise par l'agriculteur. INRA, Laon, (France). 216 p.

Bottinelli, N., Angers, D.A., Hallaire, V., Michot, D, Le Guillou, C., Cluzeau, D., Heddadj, D. and Aubry, S.M. 2017. Tillage and fertilization practices affect soil aggregate stability in a Humic Cambisol of Northwest France. Soil and Tillage Research 170: 14-17

Chenu, C., Le Bissonnais, Y. and Arrouays, D. 2000. Organic matter influence on clay wettability and soil aggregate stability. Soil Science Society of American Journal 64: 1479-1486.

Consentino, D., Chenu, C. and Le Bissonnais Y. 2006. Aggregates stability and microbial community dynamics under drying-wetting cycles in a silt loam soil. Soil Biochemistry 38(8):2053-2062.

C.P.C.S. 1967. Classification des sols. Commission de pédologie et de 
classification des sols. ENSA-Grignon; Laboratoire de pédologie-géologie. $87 \mathrm{pp}$.

Duval, M., Angers, D.A. et Laverdière, M.R. 1993. Revue de quelques facteurs régissant l'état et la stabilité de la structure du sol. Agrosol VI (2):44-51.

Fallavier, P., Babre, D. et Breysse, M. 1985. Détermination de la capacité d'échange des sols tropicaux acides. Agronomie. Tropicale 40:298-308.

FAO. 2006. World Reference Base for soil resources 2006. A framework for international classification, correlation and communication. FAO, World Soil Resources Reports $N^{\circ} 103$, Rome, Italy. $128 \mathrm{p}$.

Gelaw, A.M., Singh, B.R. and Lal, R. 2013. Organic carbon and nitrogen associated with soil aggregates and particle sizez under different land uses in Tigray Northern Ethiopia. Land Degradation and Development 26 : 690-700.

Gnankambary, Z., Sédogo, M.P., Hien, V. et Lompo, F. 1999. Détermination du facteur de correction de la méthode Walkley et Black pour le dosage du carbone organique dans les sols ferrugineux tropicaux du Burkina Faso. In 'Communication aux 5è Journées annuelles', Niamey. (Ed.). SOAChim, Ouagadougou, Burkina Faso, 5 $\mathrm{p}$.

Godefroy, J. et Jacquin, F. 1975. Relation entre la stabilité structurale des sols cultivés et les apports organiques en conditions tropicales; comparaison avec les sols forestiers. Fruits 30:595-612.

Igwe, C.A. and Nwokocha, D. 2006. Soil organic matter fractions and microaggregation in a Ultisol under cultivation and secondary forest in south-eastern Nigeria. Australian Journal of Soil Research 44: 627-635.

Jenkinson, D.S. and Powlson, D.S. 1976. The effects of biocidal treatments on metabolism in soil. V. A method for measuring soil biomass. Soil Biology and Biochemistry 8:209-213.
Kemper, W.D. and Rosenau, R.C. 1986. 'Aggregate stability and size distribution. In: Methods of soil analysis. Part 1. Physical and Mineralogical Methods, Agronomy Monograph No. 9. Klute A. (Ed.). American Society of Agronomy and Soil Science Society of America, Madison, USA,421-442.

Kouakoua, E., Larré-Larrouy, M.C., Barthès, B., De Fretas, P. L., Neves, C., H.S.G. et Feller, C. 1999. Relations entre stabilité de l'agrégation et matière organique totale et soluble à l'eau chaude dans les sols ferralliques argileux (Congo, Brésil). Canadian Journal of Soil Science 79: 561569.

Lamar, R. et Bresson, L.M. 1989. Etat d'agrégation et rôle du fer dans les horizons fersiallitiques: analyse de données. Science du Sol 27:243-256.

Le Bissonnais Y. 1988. Comportements d'agrégats terreux soumis à l'action de l'eau: Analyse des mécanismes de désagrégation. Agronomie 8:915-924.

Neufeldt, H., Ayarza, M.A., Resck, D.V. S. and Zech, W. 1999. Distribution of waterstable aggregates and aggregating agents in cerrado oxisols. Geoderma 93: 85-97.

Oades, J.M., Kirkman, M.A, and Wagner, A.G. 1970. The use of gas-Liquid chromatography for the determination of sugars extracted from Soils by Sulfuric Acid. Soil Science. Society of America Proceeding 34: 230-235.

Oades, J.M. 1984. Soil organic matter and structural stability: Mechanisms and implications for management. Plant and Soil 76: 319-337.

Ouattara, B., Ouattara,, K., Serpantié, G., Mando, A., Sédogo,, M.P. and Bationo, A., 2006. Intensity cultivation inducedeffects on soil organic carbon dynamic in the Western Cotton area of Burkina Faso. Nutrient Cycling in Agroecosystem 76:331339.

Ouattara, B., Ouattara, K., Lompo, F., YaoKouamé, A. et Sédogo, M.P. 2011. De la 
culture itinérante à la culture permanente: impact sur le statut organique et l' agrégation d'un sol ferrique à l' Ouest du Burkina Faso. Agronomie Africaine 23(1):1-9.

Ouattara, K., Ouattara, B., Nyberg, G., Sédogo, M.P and Malmer, A. 2007. Ploughing frequency and compost application effets on soil infiltrability in a cotton-maize (Gossypium hirsutum-Zea mays L.) rotation system on a Ferric Luvisol and a Ferric Lixisol in Burkina Faso. Soil and Tillage Research 95: 288-297.

Ouattara, K., Ouattara, B., Nyberg, G., Sédogo, M.P. and Malmer, A. 2008. Effects of ploughing frequency and compost on soil aggregate stability in a cotton-maize (Gossypium hirsutum-Zea mays L.) rotation system in Burkina Faso. Soil Use and Management 24:19-28.

Rouiller, J., Souchier, B., Bruckert, S., Feller, C., Toutain, F. et Vedy, J.C. 1994. Méthodes d'analyses des sols. In: Constituants et Propriétés du sol. Bonneau, M. et Souchier, S. (Eds.). Masson, Paris, France. pp. 623-631.

Sall, S.N., Masse, D.; Diallo, N.H., Sow, T.M.B., Hien, E. and Guisse, A. 2016. Effects of residue quality and soil mineral $\mathrm{N}$ on microbial activities and soil aggregation in a tropical sandy soil in Senegal. European Journal of Soil Biology 75:62-69.

Sédogo, M.P., Ouattara, B. et Lompo, F. 1994. Le carbone et l'azote dans les différentes fractions granulométriques d'un sol ferrugineux tropical : effets de quatre types d'amendements organiques. Science et Technique 21:114-124.

Six, J., Bossuyt, H., Degryze, S. and Denef, K. 2004. A history of research on the link between (micro) aggregates, soil biota, and soil organic matter dynamics. Soil and Tillage Research 79(1):7-31.
Six, J. and Paustian, K. 2013. Aggregateassociated soil organic matter as an ecosystem property and a measurement tool, 2013. Soil Biology and Biochemistry 68: 4-9.

Six, J., Feller, C., Denef, K., Ogle, S.M. and De Moraes Sa, J.C. 2002. Soil organic matter, biota and aggregation in temperate and tropical soils- Effects of no-tillage. Agronomie 22:755-775.

Srinivasarao, C.H., Venkateswarlu, B., Lal, R., Singh, A.K., Kundu, S., Vittal, K.P.R., Patel, J.J. and Patel, M.M. 2014. Long term manuring and fertilizer effects on depletion of soil organic carbon stocks under pearl millet cluster bean castor rotation in western india. Land Degradation and Development 25(2):173-183.

Tessier, D., Bigore, F. et Bruand, A. 1999. La capacité d'échange cationique : outil de prévision des propriétés physiques des sols. Compte-Rendus de l'Académie. d'Agriculture de France 56:37-46.

Throckmorton, H.M., Bird, J.A., Monte, N., Doane, T., Firestone M.K. and Horwarth, W.R. 2015. The soil matrix increases microbial C stabilization in temperate and tropical forest soils. Biogeochemistry 122 (1):35-45.

Tisdall, J.M. and Oades, J.M. 1982. Organic matter and water-stable aggregates in soils. Journal of Soil Science 33:141-163.

Wahl, N.A., Bens, O., Buczko, U., Hangen, E. and Hüttl, RF. 2004. Effects of conventional and conservation tillage on soil hydraulic properties of a silty-loamy soil. Physics and Chemistry of the Earth, Parts A/B/C 29 (11-12):821-829.

Zhao, J. Chen, S., Hu, R. and Lia, Y. 2017. Aggregate stability and size distribution of red soils under different land uses integrally regulated by soil organic matter, and iron and aluminum oxides. Soil and Tillage Research 167:73-79. 